Trayectorias Universitarias, 6(11), e033, 2020

ISSN 2469-0090 | https://doi.org/10.24215/24690090e033

https://revistas.unlp.edu.ar/TrayectoriasUniversitarias

Universidad Nacional de La Plata

La Plata | Buenos Aires | Argentina

\title{
Temas transversales en acción
}

\section{Cross-cutting themes in action}

\author{
Stella Maris Abate* \\ https://orcid.org/0000-0002-4819-5164 \\ smabate@ing.unlp.edu.ar \\ Universidad Nacional de La Plata | Argentina \\ Verónica Orellano** \\ https://orcid.org/0000-0001-8324-530X \\ orellanovero@gmail.com \\ Universidad Nacional de La Plata | Argentina
}

\section{RESUMEN}

El trabajo presenta un paneo de distintas denominaciones que han realizado colegas del campo de la pedagogía y del curriculum sobre la idea de temas transversales y controversiales en la educación. Se presentan seis temas transversales con potencia de ser abordados en clave controversial, los mismos surgen de conversaciones con colegas del campo curricular y con docentes de nuestra universidad sobre distintas propuestas "en acto". Asimismo se listan a modo de ejemplos espacios curriculares con apertura a alojar estos nuevos temas. Se cierra con una enumeración de acontecimientos y sujetos que impulsan la construcción de los temas presentados.

\section{ABSTRACT}

The work presents a survey of different denominations made by colleagues in the field of pedagogy and the curriculum on the idea of transversal and controversial topics in education. Six transversal topics are presented with the potential to be approached in a controversial code; they arise from conversations with colleagues from the curricular field and with teachers from our university about different proposals "in action". Likewise, curricular spaces opening house these new topics are listed as examples. It closes with a list of events and subjects that drive the construction of the topics presented.

\section{PALABRAS CLAVE}

Temas transversales, Temas Controversiales, Currículum en acción

\section{ABSTRACT}

Transversal topics, Controversial topics, Curriculum in action 


\section{PRESENTACIÓN}

En el presente artículo ponemos en diálogo textos sobre temas transversales y controversiales en el curriculum universitario producidos en el marco de conversaciones con docentes y colegas en los años 2018 y $2019^{i}$.

Si bien la inclusión de estos temas irrumpe como una preocupación contemporánea en el curriculum universitario, desde ámbitos afines del campo pedagógico se han realizado contribuciones valiosas que permiten dotar de mayor espesor al tratamiento de la transversalidad. Nombrados como problemas sociales relevantes (Funes y Jara, 2015), o cuestiones socialmente vivas (Legardez y Simonneaux, 2006; Legardez, 2016) entre otras $\mathrm{ii}^{2}$, dichos aportes nos permiten dar cuenta de la amplia cobertura desplegada en torno al tema que nos ocupa.

De la mano del diagnóstico de insuficiencia del curriculum como sumatoria de asignaturas, la integración curricular a través de una idea, tema o supra asignatura a la que se subordinan los diversos contenidos ha sido una preocupación recurrente en la búsqueda de alternativas.

Para avanzar en el tratamiento de recorridos formativos que favorecen el desarrollo de miradas críticas e integrales urge, en compañía de otras intervenciones, imaginar estructuras más flexibles.

Asimismo, el tratamiento transversal de saberes y temas en el curri-

i De las conversaciones participaron el Director de la Lic. y Prof. Sociología Sebastián Benítez Largui, la Directora de la Lic. y Prof. En Historia, Laura Lenci, la docente de la Lic. y Prof. en Geografía María Cecilia Zappettini, el Director de la Lic. y Prof. en Ciencias de la Educación, Martín Legarralde y la Profesora Titular del Taller Vertical C de la Lic. y Prof. en Diseño y Comunicación Visual, de la Facultad de Artes, Sara Guitelman. Asimismo se recuperan voces de colegas del campo del curriculum y de la ingeniería relevadas y sistematizadas en el marco del Proyecto de Investigación: "Temas transversales y controversiales en la formación de ingenieros: Construcción de una agenda propia"(2016-2019)".

2 Otras ideas que dan cuenta de esta preocupación pueden encontrarse en Toledo Jofré M. I., Magendzo Kolstrein A., Gutiérrez Gianella V., Iglesias Segura R. y López Facal, R. Enseñanza de "temas controversiales" en el curso de historia, desde la perspectiva de los estudiantes chilenos. Revista de Estudios Sociales [Online], 52 | Abril 2015, posto online no dia 01 abril 2015, consultado o 29 abril 2020. URL: http://journals. openedition.org/revestudsoc/9077. Además el tema ha sido abordado por autores como L. Stenhouse, H. 
culum implica reconocer interacciones transversales entre los sujetos implicados, lo que requiere escuchar y establecer vínculos con redes de docentes y profesionales preocupados en estos temas. Producto de estos vínculos hemos ido recogiendo experiencias y reflexiones que en este artículo compartimos en clave de "puestas en acto" para ubicarnos en un escenario de posibilidades que dan cuenta del lugar estratégico de los temas y saberes transversales controversiales en la formación universitaria de profesionales.

\section{ALGUNAS REFERENCIAS I}

Hemos definido los temas transversales como aquellos temas que son controversiales en tanto implican visiones en tensión sobre el abordaje de las problemáticas sociales. Para ello el aporte de Alicia de Alba (2015) al curriculum universitario es clave en tanto vincula lo controversial a los saberes transversales a la construcción política del curriculum, acentuando el papel de los transversales en su potencialidad de incorporar la direccionalidad contenida en los contornos sociales como intentos de configurar nuevos rumbos a las sociedades actuales.

De igual forma, hicimos uso de lo propuesto por Abraham Magendzo (2016), quien considera que el problema de la controversia se da al pensar el currículum en su vínculo con la sociedad y las diferentes visiones que existen en ella sobre determinados temas. La controversia en el currículum implica abordar transversalmente temas sobre los que la población tiene visiones dilemáticas y opuestas, y sobre los que se proponen explicaciones o soluciones conflictivas.

Así el concepto de cuestiones socialmente vivas trabajado por los franceses Legardez y Simonneaux (2006) focaliza en aquellos asuntos portadores de incertidumbres, divergencias, controversias, disputas, incluso conflictos.

\section{La controversia en el currículum implica abordar transversal- mente temas sobre los que la población tiene visiones dilemá- ticas y opuestas, y sobre los que se proponen explicaciones o soluciones conflictivas.}

En esa línea consideran ciertos matices en torno a la actualidad de dichos temas en el sentido de ser propios de un tiempo presente. Legardez $(2006 ; 2016)$ apunta que ello no siempre es así ya que pueden permanecer vivos durante largos períodos de tiempo, con distinta intensidad y en diferentes sectores de la sociedad. De allí que propone pensar la potencialidad de ciertos temas para devenir candentes.

Aquí nos interesa destacar la contribución que en nuestro país realizan Funes y Jara (2015) quienes acentúan su construcción en clave 
local y presente en tanto se trata de problemas articulados a la realidad social de la vida cotidiana, sin desconocer el mundo global, los pasados y la proyección a futuros.

Desde estas referencias consideramos que abordar los temas transversales (sociales y controversiales) en cualquier carrera profesional es importante porque su tratamiento permite abordar la práctica profesional desde una perspectiva amplia. La práctica profesional no solo es parte y producto de la actividad técnica que representa, además expresa modos de diálogos éticos con el contexto sociocultural en la que se desarrolla.

Hacerles un lugar explícito en el currículum universitario es posible en la medida que las instituciones los alojen en sus proyectos, compartiendo horizontes formativos preocupados en posicionar la formación de profesionales en los debates sobre los problemas sociales y económicos de la región.

\section{PROPUESTAS CURRICULARES EN ACCIÓN II}

¿Es posible acordar una agenda de temas y espacios curriculares que jerarquicen la formación social y humana de los profesionales? Motivadas por esta pregunta repasamos aquellos temas con posibilidad de devenir transversales en carreras universitarias.

Producto de conversaciones con referentes de seis carreras que se dictan en el ámbito de la UNLP, recogemos aquí experiencias y reflexiones sobre cuestiones emergentes del contexto, al modo de saberes transversales que impactan en dichas carreras.

Asimismo damos cuenta de espacios con apertura curricular para alojar estos temas, en clave de "puestas en acto o temas en acción" atendiendo al lugar estratégico de estos temas en la formación universitaria de profesionales.

\section{II. a) Temas potencialmente controversiales}

\section{II. a 1) Rol del estado y (des)vinculaciones entre universidad y estado}

Lo público y la universidad como espacio público es otro de los temas que se podrían presentar como controversial en tanto se tensionan visiones sobre la universidad acompañando al estado o construyendo un discurso propio. 
En la carrera de Ciencias de la Educación se visualiza un posible campo de controversias en relación con formas de pensar la educación popular, que a fines de los ochenta principios de los noventa del siglo pasado tenía un componente crítico sobre la escuela. Sin embargo, la reforma educativa que se pone en marcha a partir de 1992, cancela o suspende las controversias que venían siendo exploradas en el proceso de la transición democrática. En este sentido se produciría una especie reducción del campo de controversias posibles, una suerte de reacción defensiva frente a la lectura de cómo la reforma educativa avanzaba en claves más tecnocráticas; es decir diferencias de perspectivas, de enfoques, de temáticas son postergadas por la necesidad de defender la escuela.

Del mismo modo ocurre con el protagonismo otorgado a la ciencia y la tecnología en el desarrollo de nuestro país. Apoyados en argumentos como el que expresa Hurtado se despliegan distintas propuestas. El doctor en Física sostiene que siempre que países como Argentina se proyectaron como naciones emergentes, los poderes centrales desarrollaron estrategias para frenar su progreso $\mathrm{iii}^{3}$.

La cuestión Malvinas es un ejemplo de tema en agenda en la UNLP, en tanto condensa discursos que promueven una perspectiva descoIonizadora en muchos sentidos: territorial, usos de los recursos, producción de ciencia y tecnología.

Por otro lado, en vínculo con la economía se puede hablar de diferentes roles del Estado en la creación de condiciones para el desarrollo económico y el progreso social. En conversación con Daniel Feldman, a propósito de la relevancia de este tema en la formación de ingenieros, se destaca la tensión entre un contexto en el que se contraponen visiones liberales que conciben al Estado asegurando que prosperen las iniciativas privadas, o visiones desarrollistas que conciben al Estado como un agente de cambio e innovación. La perspectiva del desarrollo nacional empieza a formar parte en la formación de ingenieros: poder entender los escenarios actuales en términos de la historia económica del país y de la confrontación entre los modelos de país exportador primario versus los proyectos industrialistas y productivos resultaría esencial para situar la formación en coordenadas reales.

\section{II. a. 2 Problemática ambiental y modelos de desarrollo}

Distintos colegas del campo curricular consultados destacan que se trata de un tema de mayor carácter comprensivo en tres sentidos: a) refiere a una problemática crucial del mundo contemporáneo en cuya dilucidación se juega el futuro de nuestras sociedades, al tiempo que repercute comprometiendo gravemente las condiciones de vida

3 Diego Hurtado. "Cuando la ciencia se hace política" Entrevista publicada en Página 12 el 19 de julio de 2017. Disponible en https://www.pagina12.com.ar/51006-cuando-la-ciencia-se-hace-politica 
de distintas clases y sectores sociales, etnias, género y grupos etarios; b) involucra demandas de actores con intereses distintos: las empresas ocupadas en la rentabilidad, el gobierno en busca de productividad y desarrollo económico, comunidades epistémicas en la defensa de sus conocimientos y la sociedad en busca de calidad de vida; $\mathrm{y}$ c) otros temas como el problema de la soberanía alimentaria y las prácticas socio-comunitarias tendrían cabida en la problemática ambiental y requieren no sólo investigación sino también tratamiento por vías del currículo ${ }^{4}$

Discusiones alrededor de modelos económicos lineales o economías circulares asoman en el escenario universitario sobre ideas de progreso y desarrollo económico. En variados espacios de intercambio se identifican discursos acerca del valor de los productos, los materiales y los recursos; los impactos en el ambiente y la inclusión de distintos sectores sociales en la mano de obra y en el consumo de lo producido.

En Ingeniería es un tema de tratamiento y la universidad apoya, con distintas unidades académicas, prácticas concretas de economías que hacen eje en la producción y comercialización solidaria. Además motiva discusiones ya que se piensa e interviene en los problemas ambientales desde concepciones variadas respecto a qué es el ambiente, si es condición de borde o parte inherente de los problemas que abordan estos profesionales y cuáles son las voces legítimas para ocuparse del tema. También es objeto de controversia en cuanto se lo trate sólo como una cuestión técnica-instrumental o además como un asunto político, ya que para abordarlo en su complejidad es preciso discutir qué modelo de desarrollo económico (y tecnológico) se propone a nivel global, y si el mismo supone para su existencia la desigualdad social y económica, estrechamente vinculada a los problemas ambientales.

\section{II. a. 3 Derechos humanos, género y diversidad}

Hay cierto consenso en las carreras de la UNLP en el reconocimiento de los derechos humanos y sociales como un marco desde el cual algunas cuestiones se vuelven controversiales.

Por una parte se trata de un tema de agenda de la UNLP, materializado en variados espacios de gestión y académicos (Prosecretaría de Derechos Humanos, Instituto de Derechos Humanos de la JURSOC, así como Diplomatura de Género y Derechos Humanos, etc.)

Ya sea por el peso de las prioridades políticas respecto del lugar que los derechos humanos tienen para la formación de los estudiantes universitarios, o traccionado por la inclusión de estos temas en los diseños curriculares de la educación obligatoria, referencia para los estudiantes que ejercen posteriormente la enseñanza, los derechos humanos de algún modo comienzan a atravesar distintas materias de la carrera de Historia de la FaHCE. 
Bajo la idea de que ninguna controversia se despliega como tal de manera aislada, sino que adquiere sentido en la medida en que se relaciona con otras cuestiones problemáticas, constituyendo una especie de tejido o red (Nudler, 2009), los derechos humanos potencian su posibilidad controversial en diálogo con otras perspectivas como la perspectiva de género.

Bajo la idea de que ninguna controversia se despliega como tal de manera aislada, sino que adquiere sentido en la medida en que se relaciona con otras cuestiones problemáticas, constituyendo una especie de tejido o red (Nudler, 2009), los derechos humanos potencian su posibilidad controversial en diálogo con otras perspectivas como la perspectiva de género.

En este sentido cuenta con profusos estudios en el ámbito y que goza, junto al tema de la discapacidad, de un tratamiento transversal en los proyectos institucionales: desde el debate sobre el uso del lenguaje inclusivo hasta la mirada sobre los sujetos destinatarios de la acción profesional, es decir, desde y para qué sujeto se concibe la obra que por lo general tenía como destinatario un sujeto universal.

\section{II.a.4. Formar un intelectual crítico o un profesional libre.}

Lo ontológico de las profesiones es controversial y da apertura a la controversia. La constitución de profesiones y disciplinas como el caso de la sociología es controversial porque hay interpretaciones sobre los temas transversales: no hay un proceso de clausura en la que todos se pongan de acuerdo acerca cómo interpretar la desigualdades, las relación de poder, los modos de acumulación, los procesos de reproducción, legitimación y cambio social.

\section{La constitución de profesiones y disciplinas como el caso de la sociología es controversial porque hay interpretaciones sobre los temas transversales: no hay un proceso de clausura en la que todos se pongan de acuerdo acerca cómo interpretar la des- igualdades, las relación de poder, los modos de acumulación, los procesos de reproducción, legitimación y cambio social.}

Esta tensión entre perfiles más profesionales o disciplinares se expresa en el perfil de egreso de la carrera de Sociología; si bien sería una discusión que se va saldando hay huellas de esta tensión entre la formación de un intelectual crítico o un profesional que pueda actuar en público y en lo privado como un profesión libre, discusión que en 
los años sesenta y setenta se expresaba entre academicistas y profesionistas. En el siglo actual la inserción de los sociólogos en el estado y la definición de política públicas contribuye a que sean consideradas voces legitimadas, a pesar de cierta representación del sociólogo como un profesional que sabe manejar estadísticas pero al que no se le reconocen otros saberes.

También en carreras proyectuales esta tensión se expresa en términos de primacía de la función vs. lo estético y la alternativa marketing o arte comprometido con la cultura. En este sentido la relación entre el diseño y el arte es una controversia constitutiva de la carrera diseño en comunicación visual, cuya discusión hoy se ha revitalizado.

En la actualidad se habla de la user experience. Esta visión hizo que el diseño se vincule a lo científico y a lo técnico y se desvincule del arte. En la gran mayoría de las instituciones donde se enseña hoy el diseño prevalece el marketing y una teoría de la comunicación complaciente con las formas que propone el mercado. De modo alternativo el vínculo con el arte la aproxima a posiciones críticas, humanísticas y a la idea de sujeto creador. Esta separación del diseño con el arte es un tema controversial toda vez que hace al objeto y enfoque de la profesión en Diseño y Comunicación Visual.

\section{II. a. 5 Relación de los saberes disciplinares con los saberes pedagógicos.}

Una preocupación sobre el rol de la pedagogía en el curriculum universitario consiste en cómo entrar en diálogo con otros discursos disciplinares. Si bien esta preocupación es tematizada por diversos y diversas colegas sigue siendo válida la pregunta que se realizan Adela Coria y Gloria Edelstein (1993) acerca de si es posible el discurso pedagógico en el ámbito universitario.

En otro trabajo hemos podido analizar el caso de la intervención pedagógica en carreras científico tecnológicas como lo es la Ingeniería. "En este sentido, la incorporación de saberes sociales y humanísticos como estándar constituyó una oportunidad para implicarse desde la intervención docente, ya que habilitó un escenario para que estos saberes se constituyan en objeto de enseñanza." (Abate y Lucino, 2017, p. 76)

En cuanto a carreras sociales y humanísticas, a pesar de una mayor cercanía, la valoración de la especificidad disciplinar de la pedagogía es una deuda pendiente. Enseñar a otros a enseñar requiere invitar a otros a prácticas de imitación (alejadas del buen hacer artesanal) resumida en expresiones como, explico los temas como ellos deberían hacerlo en el secundario. Habría aquí una idea de enseñanza como explicación y cierta resistencia a valorar el contexto como traductor de saberes y otorgador de sentidos.

En general la Pedagogía es representada como un saber de "los 
cómo" y las decisiones sobre los contenidos del dominio exclusivo de cada disciplina; es percibida entonces como un saber extranjero que no participa de las discusiones en torno a qué enseñar. Por otra parte y en clave de pensar esta relación, en los últimos tiempos ciertos usos de las tecnologías digitales vinieron a opacar las discusiones pedagógicas.

En síntesis, las cuestiones emergentes son aquellas que se convierten en algo necesario de pensar, de discutir, de problematizar; son aquellos problemas de la época que podrían originar temas transversales y controversiales en la formación de profesionales. Además aparecen controversiales históricos o que hacen a la constitución de la disciplina ya sea como marca de origen o como sedimento de ciertas discusiones que se dan a lo largo de la historia de la carrera o de cómo se ha ido pensando la formación de profesionales y que se reeditan o no en ciertos momentos.

\section{II b) Espacios curriculares con apertura para alojar estos temas}

Activar espacios que promuevan procesos de cambio en las carreras universitarias conlleva una complejidad que no desconocemos; sobre todo cuando se trata de modificar o poner en cuestión las jerarquías hegemónicas de los saberes válidos a enseñar. No obstante la primacía de los formatos disciplinares en el curriculum de nuestra universidad, vale la pena identificar posibles espacios curriculares e institucionales que den entrada a lo contingente y lo provisorio.

En líneas generales se observan propuestas de soluciones más canónicas desde el punto de vista disciplinar que le asignan a estos temas espacios curriculares estables y específicos, o proponen que anclen en asignaturas electivas, seminarios y talleres distribuidos transversalmente a lo largo de los planes de estudio. Centralmente estas soluciones se jerarquizan o legitiman desde la norma (estándares, cambios de planes de estudio) y desde la posibilidad de que se impulsen desde campos académicos reconocidos como tales.

Otras proponen espacios más flexibles aunque en el borde de lo establecido y permiten la entrada y salida periódica de temas o habilitan la irrupción en el currículum de acontecimientos sociales relevantes para la formación de profesionales. Estas soluciones se legitiman en conversación de la universidad con los acontecimientos sociales, los territorios en los que ancla la formación o los movimientos sociales e implican que docentes, estudiantes y comunidad participen en la definición de los contenidos de la formación, conversando en contextos de relaciones de poder.

Para el caso de Ciencias de la Educación las posibilidades de que el horizonte de ciertos acontecimientos o procesos históricos impacten en el curriculum se ubican más allá del texto curricular que supone mayor estabilidad. 
El plan de estudio de Ingeniería contempla en sus planes de estudio materias humanísticas, más estables, y complementarias, ofertas más móviles como primeras entradas a estos temas.

En cuanto al plan de estudios de Historia uno de los espacios que se destaca con mayor apertura para incluir este tipo de temas son las "materias problemas". En el decir de sujetos claves que participaron en su ideación constituye una solución curricular incluir materias organizadas en torno a problemas y controversias disciplinares de "hechos, fenómenos, procesos".

Por su parte el Taller de Diseño en Comunicación Visual C, de la Facultad de Artes, UNLP, incorpora en 2001 la "extensión" a sus contenidos curriculares a través de un programa que se llama desde entonces "Diseño activo", y que se sustenta en el modelo de la educación experiencial. En aquel momento, en el contexto de la crisis económica nacional, la cátedra se planteó la necesidad de participar activamente con los alumnos en manifestaciones protesta social, desde el diseño como práctica discursiva. (Guitelman, Abate; 2013)

También el espacio de Cátedras Libres de la Universidad, ha sumado propuestas orientadas al estudio de temas controversiales en los últimos años.

\section{A modo de cierre}

Cerramos este artículo señalando que la aparición de ciertos temas controversiales va de la mano de actores que ponen en debate la interpretación sobre estos temas y problemas transversales.

En las licenciaturas de Historia, Educación y Geografía las controversias van de la mano de ciertos procesos, de grandes transformaciones sociales y periodos de crisis y emergencias de nuevos actores.

Tanto en estas carreras como en Sociología, los estudiantes y graduados son considerados impulsores de ciertos cambios; en este último caso a partir de la demanda por la inclusión de temas como la perspectiva decolonial, los saberes sociales y políticos desde la perspectiva de pueblos originarios y de los teóricos latinoamericanos pone en tensión la predominancia de la teoría social anglosajona, latino europea y norteamericana.

En el caso de los profesorados, los Diseños Curriculares de la Provincia de Buenos Aires son también fuentes de cambio y en este sentido podemos decir que el Estado tracciona cambios en la formación universitaria. Por ejemplo el diseño curricular de la Provincia de Buenos Aires del 2006 introduce nuevos temas de las geografías posmodernas, humanistas, radicales y lo que se llaman en la actualidad las "otras geografías", es decir, iba delante de la formación universitaria para la enseñanza de la geografía.

La existencia de Abuelas de Plaza Mayo, los Movimientos sociales y 
los Movimientos feminista y la Ley Nacional № 27499 han traccionado a generar un marco para la generación de agenda.

El Instituto Malvinas dependiente de la Facultad de Ingeniería de la UNLP es un espacio generador de sentidos nacionales. Los estándares de acreditación de las diferentes carreras propuestos por Coneau y sus distintas organizaciones disciplinares constituyen también impulsos de cambio.

\section{El Instituto Malvinas dependiente de la Facultad de Ingeniería de la UNLP es un espacio generador de sentidos nacionales. Los estándares de acreditación de las diferentes carreras propues- tos por Coneau y sus distintas organizaciones disciplinares cons- tituyen también impulsos de cambio.}

El tema tratado en este artículo toma forma comunicacional en un tiempo de pandemia. Caracterizado como un tiempo extraordinario, como una situación sin precedentes, las ciencias sociales califican a esta pandemia como un "hecho social total", en el sentido de que convulsiona el conjunto de las relaciones sociales, y conmociona a la totalidad de los actores, de las instituciones y de los valores. (Ramonet, 2020) .

Seguramente a la distancia de este presente excepcional, lo dicho podrá ser reescrito al ritmo de nuevas conversaciones, oportunidades y acontecimientos. 
Abate, S. M. (Comp.) (2020). "Textos curriculares en contexto. Saberes, sujetos e instituciones". Edulp. Disponible en http://sedici.unlp.edu.ar/handle/10915/105001

Abate, S. M.; Lyons, S.; Orellano, V. (2019). "Temas transversales en el curriculum universitario: abriendo posibilidades. Miradas en conversación". Archivos de Ciencias de la Educación, 13 (15) Disponible en: http://www.memoria.fahce.unlp.edu.ar/art_revistas/pr.10929/pr.10929.pdf

Abate, S. M.; Lyons, S.; Lucino, C. V.; Giuliano, G. (2019). “Propuestas curriculares para contenidos transversales (sociales y controversiales) en carreras de ingeniería". V Jornadas de Investigación, Transferencia y Extensión de la Facultad de Ingeniería, La Plata. Disponible en http://sedici.unlp.edu.ar/handle/10915/75013

Abate, S. M.; Lucino, C. (Coord) (2017). "Ingeniería y saberes sociales. Diálogos posibles". EDULP. Disponible en http://sedici.unlp.edu.ar/handle/10915/61860

Coria, A.; Edelstein, G. (1993). "El pedagogo: un discurso posible". Pensamiento universitario. Año 1 (1) FCS. Buenos Aires.

De Alba, A. (2015). "Cultura y contornos sociales. Transversalidad en el currículum universitario". En A. de Alba y A. Casimiro Lopes (Coords.) Diálogos curriculares entre México y Brasil (195-211) México: IISUE.

Guitelman, S. Abate, S. “Diseñadores ¿para qué?”. Argentina. San Luis. 2013. Encuentro. Séptimo Encuentro Nacional y Cuarto Latinoamericano "La universidad como objeto de investigación". Universidad Nacional de San Luis

Legardez, A. et Simonneaux, L. (2006). “'école à l'épreuve de l'actualité - Enseigner les questions vives". Paris: ESF

Legardez, A. (2016). “Questions Socialement Vives, et Education au Développement Durable". L'exemple de la question du changement climatique. Revue francophone du développement durable (ClermontFerrand) https://hal-amu. archives-ouvertes.fr/hal-01794781/document

Funes, G.; Jara, M. (2015). "La enseñanza de las Ciencias Sociales, la Historia, la Geografía y los problemas sociales". En Funes, G.; Jara, M. Historia y Geografía. Propuestas de Enseñanza. Universidad Nacional del Comahue Facultad de Ciencias de la Educación. Educo ISBN: 978-987-33-9342-6 FACE, Cipolletti https://apehun.uncoma.edu.ar/index.php/publicaciones/24-de-ensenanza 
Magendzo, A. (2016). "Incorporando la perspectiva controversial en el currículum disciplinario". Revista Iberoamericana de Educación Superior (RIES) 12(19).

\section{$\mathrm{CV}$}

*Prof. en Ciencias de la Educación (UNLP). Magister en Educación (Orientación Ciencias Sociales)- UNICEN

Profesora Adjunta en la Cátedra Teoría y Desarrollo del Currículum - Carrera de Ciencias de la Educación. Facultad de Humanidades y Ciencias de la Educación.

Directora de proyectos de Investigación sobre los saberes sociales en carreras tecnológicas en el marco del Programa de Incentivos

Docente en Seminarios y Cursos de Formación Docente en la Facultad de Ingeniería y en la Especialización en Docencia Universitaria UNLP

Coordinadora del Área Pedagógica y Prof. Adjunta en la Asignatura Ingeniería, Comunicación y Educación - Carreras de Grado - FI - UNLP.

** Prof. en Ciencias de la Educación (UNLP).

Participante en proyectos de Investigación sobre el currículum de Enseñanza Superior y Universitario.

Docente en la Cátedra Teoría y Desarrollo del Currículum - Carrera de Ciencias de la Educación. Facultad de Humanidades y Ciencias de la Educación.

Tutora docente en la Especialización en Docencia Universitaria UNLP. 